Review

\title{
Chronic Progressive Nephropathy (CPN) in the Rat: Review of Pathology and Relationship to Renal Tumorigenesis
}

\author{
John Curtis Seely ${ }^{1}$ and Gordon C. Hard ${ }^{2}$ \\ ${ }_{2}^{1}$ Experimental Pathology Laboratories, Inc., PO Box 12766, Research Triangle Park, NC 27709, USA \\ ${ }^{2}$ Private Consultant, Tairua, New Zealand
}

\begin{abstract}
Chronic progressive nephropathy $(\mathrm{CPN})$ is a rodent-specific, age-related renal disease, particularly of male rats, characterized by a spectrum of distinct histological changes which may begin early in the animal's life and progress to end-stage renal disease in certain rat strains. Although CPN-related pathology is well known to most toxicological pathologists other features of CPN such as pathogenesis, modulating factors, proliferative nature, response to chemical exposure and relationship to tumorigenesis are less clearly acknowledged. CPN is generally regarded as a degenerative to atrophic disease with compensatory regenerative hyperplasia. The proliferative nature of CPN often becomes problematic in advanced to end-stage renal disease. At this stage, a number of tubule profiles may be mistaken for atypical tubule hyperplasia, the reported precursor lesion of tubule adenoma. CPN associated proliferative tubule profiles must be carefully separated from atypical tubule hyperplasia particularly in studies where chemical exposure has exacerbated CPN. Over the past several years increasing evidence has supported the hypothesis that CPN may be regarded as a type of "mode of action" during renal carcinogenesis in rodent bioassay studies. Retrospective studies of control and treated animals have consistently shown a relationship between the increased severity of CPN and the presence of atypical tubule hyperplasia and small, incipient renal adenomas. Understanding CPN-related tumorigenesis is important for human risk assessment interpretation. Since CPN is a rodent specific disease with no apparent similar human kidney disease condition, evidence that renal tumors may arise from an interaction with CPN could assist regulatory agencies in interpreting data from studies with exacerbated CPN. (J Toxicol Pathol 2008; 21: 199-205)
\end{abstract}

Key words: rat, kidney, chronic progressive nephropathy, pathology, tumorigenesis, risk assessment

\section{Introduction and Overview}

Chronic progressive nephropathy $(\mathrm{CPN})$ is one of the most widely recognized disease entities in rodent preclinical studies. CPN is a common spontaneous age-related disease of rodent kidneys, particularly in rats ${ }^{1-3}$. Because of the kidney's central role in maintaining the normal physiologic balance of the body's internal environment and its importance in drug metabolism and excretion, understanding the basic mechanisms and pathology of CPN is critical. The etiology of CPN remains unknown. In some models of nephropathy, speculation regarding hemodynamic alterations leading to hyperperfusion and hyperfiltration of macromolecules within the glomerulus resulting in mesangial overload and glomerulosclerosis has been postulated ${ }^{4}$. However, hemodynamic alterations do not seem to be associated with $\mathrm{CPN}^{5}$. There are strain, sex, and

Received: 16 April 2008, Accepted: 29 August 2008

Mailing address: John Curtis Seely, Experimental Pathology

Laboratories, Inc., PO Box 12766, Research Triangle Park, NC, 27709,

USA

TEL: 1-919-313-0630 FAX: 1-919-998-9607

E-mail: jseely@epl-inc.com age differences with respect to the incidence and severity of CPN associated lesions ${ }^{6-10}$. Sprague-Dawley and F344 rats generally have an earlier onset and higher incidence and severity than the Wistar, Brown Norway and Long-Evans rat strains. Although the term CPN has undergone a number of name changes over the years, currently CPN or "nephropathy" are synonymous terms and recommended by standardized nomenclature classifications.

Pathologists beginning their career in toxicological pathology are quickly exposed to the spectrum of CPN associated lesions such as regenerative (basophilic) tubules surrounded by thickened basement membranes, hyaline proteinaceous casts, glomerulosclerosis, interstitial fibrosis and infiltration of mononuclear inflammatory cells which can begin as early as $2-3$ months of age and progress continually through the life-span of the animal. Histological changes associated with CPN also result in a number of functional changes that result in increased proteinuria and decreased urine concentration ability ${ }^{11,12}$.

Most pathologists agree that the earliest CPN lesion, by light microscopy, is represented by small cross sections of basophilic tubules surrounded by a thickened basement membrane in the renal cortex. Only later does another one of the hallmarks of CPN, glomerulosclerosis, become 
evident. By the end of 2-year carcinogenic studies, the advanced stage of CPN may result in end-stage kidney and death of the animal due to chronic renal failure. In fact, mortality associated with CPN is an issue with serious implications in the conduct of some carcinogenic studies ${ }^{13}$. CPN may be modulated by a number of factors including diet, administration of hormones and many other experimental manipulations ${ }^{13-17}$. Recently, the type of diet particularly with regard to the protein content and/or caloric restriction have been investigated as ways to reduce the severity or limit the progression of $\mathrm{CPN}$, thereby, extending the life of the animal ${ }^{14,18}$.

CPN has been described as a degenerative to atrophic disease with compensatory hypertrophy and hyperplasia. The proliferative rate of CPN has been demonstrated by cell labeling studies, which have shown cell proliferative rates within CPN affected tubules increased over that of normal tubules $^{19-21}$. In advanced stages of nephropathy, a number of proliferative tubule profiles may be recognized as having an increased number of lining epithelial cells. These tubule profiles are problematic for many pathologists as they may be mistaken for atypical tubule hyperplasia (ATH), the precursor lesion of renal adenoma. Hard and Seely ${ }^{22}$ recently published recommendations to assist in the differentiation of CPN tubules from actual ATH and early renal tubule adenomas. An association between CPN and the presence of ATH and renal tubule tumors (RTT) has been observed from several carcinogenic studies in which chemical administration exacerbated the severity of $\mathrm{CPN}^{23-25}$. Retrospective studies of control and treated animals have consistently shown a relationship between the increased severity of CPN and the presence of ATH and RTT. The underlying factors associated with this relationship are not known but, most likely, are multi-factorial and complex.

The interpretation of a renal tumorigenic response related to CPN is important for risk assessment analysis since apparently there is no human renal disease which is similar to CPN. Therefore, it has recently been postulated that RTT linked to CPN has no relevance for extrapolation to human risk assessment because CPN is a rodent- specific disease ${ }^{3}$.

This review was written primarily for toxicologic pathologists, especially study pathologists, who would like a concise yet instructive review of CPN with emphasis on pathology and pathology-related issues. Furthermore, this review is not intended to be a comprehensive overview of all aspects of CPN. Current investigations of CPN associated tumorigenesis are also presented. These studies have been the interest of the authors for some time. This postulated carcinogenic "Mode of Action" is unique and undoubtedly will have to undergo further examination and review by both researchers and by federal agencies responsible for chemical and drug regulation.

\section{Histological Spectrum of CPN Pathology}

As noted previously, CPN is characterized by a spectrum of histological changes including regenerative tubules (basophilic tubules) lined by a thickened basement, the presence of eosinophilic hyaline proteinacious tubule casts, glomerulosclerosis, interstitial fibrosis and infiltration of a variable mononuclear cell infiltrate ${ }^{1-3,8,17,26,27}$. It has been reported that the earliest change by light microscopy of $\mathrm{H} \& \mathrm{E}$ stained sections is the presence of one or more cortical solitary basophilic tubule cross sections surrounded by a thickened basement membrane (Fig. 1). The thickened basement membrane is one of the key components of CPN throughout the course of the disease. The presence of this change is helpful in distinguishing early CPN and regeneration due to toxic insult. If one examines $\mathrm{CPN}$ affected kidneys in detail, degenerative to apoptotic cells, as well as mitotic figures may be evident and responsible for the regenerative appearance. However, in most instances, these changes are not fully appreciated unless thorough examination under higher power is conducted. In some instances, affected tubules may appear as slightly dilated. Therefore, the tubule appearance is due to the presence of increased numbers of lining epithelial cells with basophilic cytoplasm and crowded, slightly enlarged and vesicular nuclei, all responsible for the overall basophilia noted on $\mathrm{H} \& \mathrm{E}$ staining. As the number of affected tubules increase, a minimal infiltration of mononuclear cells becomes evident within the interstitium. At this time, a few eosinophilic hyaline tubule casts may be noted in Henle's loops within the medulla. When hyaline casts are observed in the absence of basophilic tubules, early CPN is still usually suspected because affected CPN tubules in the cortex are most likely out of the plane of section. Shortly thereafter, changes within affected glomeruli may be noted. These changes reflect the early segmental and basement membrane thickening of the glomerular tuft and Bowman's capsule. Occasionally, a lightly eosinophilic staining and amorphous material may be present in Bowman's space. Variablysized, eosinophilic protein droplets in sporadic tubules may also be observed. Proteinuria or the loss of urinary protein in the urine, associated with CPN, is best determined by measuring urinary albumin excretion ${ }^{28}$. By electron microscopy, it has been reported that glomerular injury may be observed ${ }^{29}$. However, there is no clear evidence that these changes precede the earliest tubule changes.

Among pathologists, discussion regarding the earliest lesion, namely the basophilic tubules, often causes debate on the most correct terminology pathologists should use to describe this change. Some pathologists refer to all changes associated with CPN as "CPN or nephropathy" while others diagnose either "basophilic tubules" or "tubule regeneration". Because "basophilic tubules" may result from a number of differing pathogenic mechanisms, the authors do not recommend the use of "basophilic tubules" as a descriptor for the earliest lesion. However, if "basophilic tubule" is used then it is suggested that this term be defined in the narrative portion of the pathology report with regard to its association with CPN. Furthermore, it is highly recommended not to separate and grade each component of CPN individually. This effort requires considerable 
diagnostic consistency and time and often results in lengthy and confusing incidence tables which add little to the overall interpretation of a chemical effect. All of the components of CPN should be grouped together as CPN or alternately, as nephropathy.

As the animal ages, the extent within the kidney and the severity of CPN-related areas increase noticeably. Minimal changes usually result in multiple foci of CPN-affected areas randomly found throughout the cortex (Fig. 2). As the disease progresses, hyaline casts become more prominent and are seen extending down through the outer to inner medulla within Henle's loop. With increasing severity, foci of CPN-affected areas begin to merge and become confluent with each other resulting in a more diffuse change. Continued progression of CPN results in striking tubule, glomerular and interstitial changes. Foci of basophilic tubules are remarkable due to increased amounts of thickened basement membrane material. An occasional cortical tubule becomes hypertrophic with increased amounts of pale eosinophilic staining cytoplasm. Glomeruli may either appear hypertrophic or atrophic due to glomerulosclerosis and the presence of adhesions between the glomerular tuft and Bowman's capsule. The presence of interstitial fibrosis often results in tubule dilatation and/or cyst formation. This phenomenon appears to be the result of interactions between fibrogenic growth factors produced by tubule epithelial cells, macrophages and myofibroblasts ${ }^{30,31}$. In "End-Stage" CPN hyaline casts may also be seen in collecting ducts. In advanced to end-stage CPN, little normal renal cortical tissue remains (Fig. 3). Grossly, these kidneys are enlarged, pale and with a pitted surface. Animals that have died from end-stage renal disease often have diffuse mineralization of tubule basement membranes. Secondary hyperparathyroidism and mineralization of other tissues may be noted.

Additionally, in more advanced cases of CPN small irregularly-shaped, edematous and hyperplastic outgrowths of epithelium are often noted on the surface of the renal papilla (Fig. 4). These projections seemingly have little pathological significance. Vascular or hemorrhagic lesions are not a prominent part of the overall histological spectrum of CPN. On rare occasion only, the authors have noted perivascular inflammatory cell infiltrates and even some vasculitis and thrombosis. A light golden-brown pigment is often noted in CPN tubules and interstitial tissue. This pigment seems to be predominantly iron-positive and presumably hemosiderin ${ }^{32}$.

Grading the severity of CPN is mainly a subjective evaluation often based on the pathologist's training and experience. There is little published guidance on how to grade $\mathrm{CPN}^{13}$. Most $\mathrm{CPN}$ grading schemes grade $\mathrm{CPN}$ on a 3,4 or 5 grade scale, representing severities from minimal to markedly-severe (End-Stage). Although all of these grading schemes are appropriate, if used consistently, grades $1-4$ or 1-5 are more likely to help in the determination of an exacerbated chemical effect and are used more regularly on a global basis. Depending on the length of the study, study pathologists may also modify their grading scheme for more acute studies versus carcinogenicity studies. Furthermore, in any event of a potential chemical effect involving CPN, pathologists should provide a detailed description of the grading criteria for $\mathrm{CPN}$ in the narrative report. This provides information for the regulatory agencies to use in interpreting and comparing data from these studies and assists the peer review pathologist in determining the accuracy and consistency of the study pathologist when peer review is conducted.

\section{CPN Associated Cell Proliferation and Proliferative Lesions}

As previously noted, CPN has been described as a degenerative to atrophic disease with compensatory hypertrophy and hyperplasia. The prominent regenerative tubules noted with CPN are part of the compensatory hyperplastic response. The term "simple tubule hyperplasia" was introduced to denote a tubule appearance consisting of a single cell layer of increased numbers of lining epithelial cells and to help differentiate CPN tubules from ATH or, alternately diagnosed as, "renal tubule hyperplasia" 33 . Although pathologists differ in their diagnostic approach to the early changes associated with CPN, "tubule regeneration" or "basophilic tubules" are terms used more frequently. Other pathologists simply include this early change within the overall diagnosis of CPN when they are reasonably comfortable with their diagnosis. The term simple tubule hyperplasia has not been routinely used to diagnose the early tubule change ${ }^{34}$. However, the crowded nature of the lining cells of CPN-affected tubules implies the presence of simple tubule hyperplasia.

Although it was easy for pathologists to recognize the increased number of lining epithelial cells within the confines of a single tubule by routine H\&E light microscopy, it was not until sometime later when investigators began to apply specialized techniques such as tritiated thymidine autoradiography to demonstrate the cell labeling index (LI) of cells undergoing DNA synthesis within areas of $\mathrm{CPN}^{19-21}$. These studies have reported increased LI of up to 10 times greater in CPN affected areas than normal areas. It appears that the proliferation rate of affected tubules is the same for male and female rats; however, the number of affected tubules is greater in males ${ }^{17}$. Proliferating cell nuclear antigen (PCNA) may also be used to measure CPN cell proliferating activity ${ }^{35}$.

In examining kidneys from rats with advanced (moderate to marked severity) to end-stage CPN, it is common to observe tubules containing proliferative changes which exceed the normal expectation for tubule regeneration or simple tubule hyperplasia. A number of these tubule phenotypes have been problematic for pathologists often leading to inappropriate diagnoses and interpretation. However, recent guidelines have been published which offer recommendations in the interpretation of these proliferative changes associated with $\mathrm{CPN}^{22}$. In addition, detailed criteria 


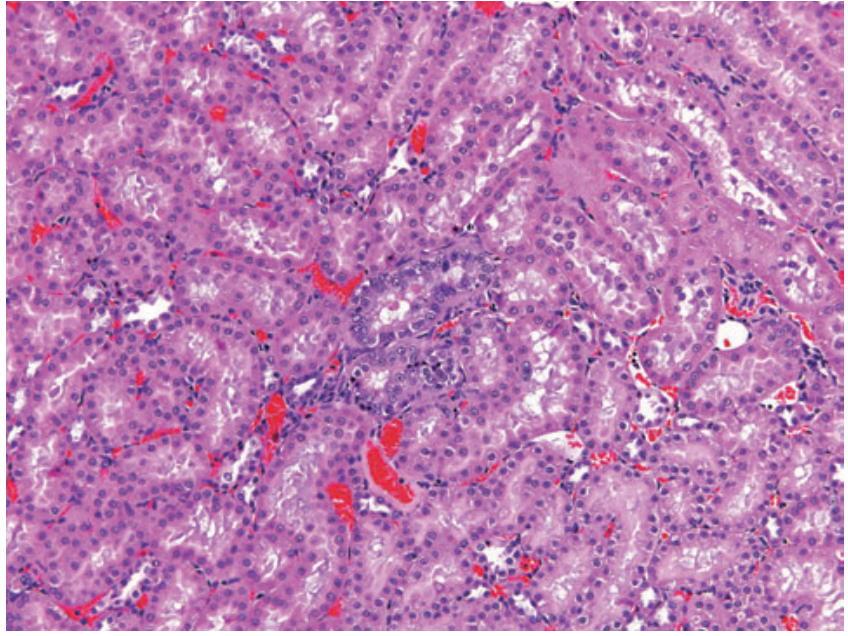

Fig. 1. Early CPN tubules characterized by regenerative changes (cytoplasmic basophilia and nuclear crowding) and thickened basement membrane. H\&E. $\times 20$.

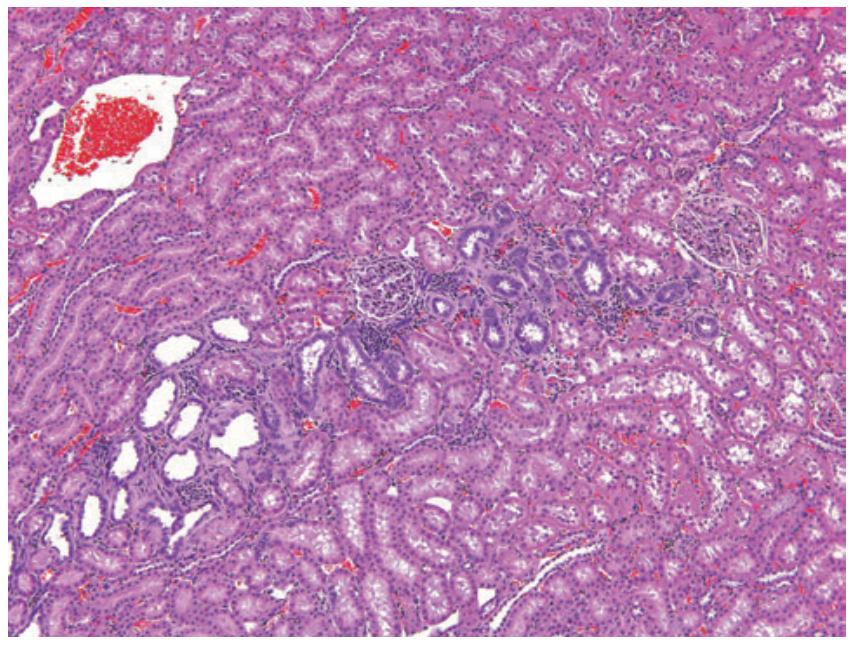

Fig. 2. Minimal, focal CPN illustrating main components of CPN; tubule basophilia, thickened basement membranes and infiltration of mononuclear inflammatory cells. Glomeruli are normal appearing. H\&E. $\times 10$.

were published in these studies to assist in the differentiation between CPN tubules and ATH. Therefore, a companion study, to the original paper by Hard and Seely, was conducted which investigated these challenging tubule profiles in more detail using special stains and serialsectioning to better understand the origin and fate of these tubules. The results from this investigation supported the proliferative nature of these tubules but none progressed outside the confines of a single tubule and they lacked the additionally reported features of ATH and, accordingly, were not considered as preneoplastic lesions ${ }^{36}$. Furthermore, the guidelines recommended by Hard and Seely ${ }^{22}$ were prepared specifically for cases involving advanced CPN in preclinical rodent studies and were not meant to be used in studies outside the context of advanced CPN. It was suggested that these proliferative tubule profiles be regarded

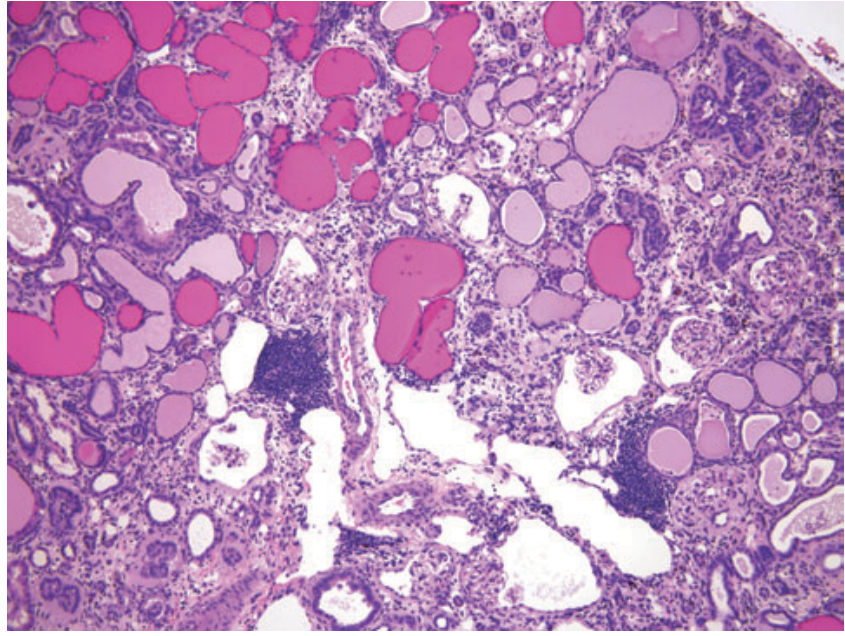

Fig. 3. Severely affected (End-Stage) CPN kidney. Entire kidney is affected and hyaline casts are prominent. H\&E. $\times 10$.

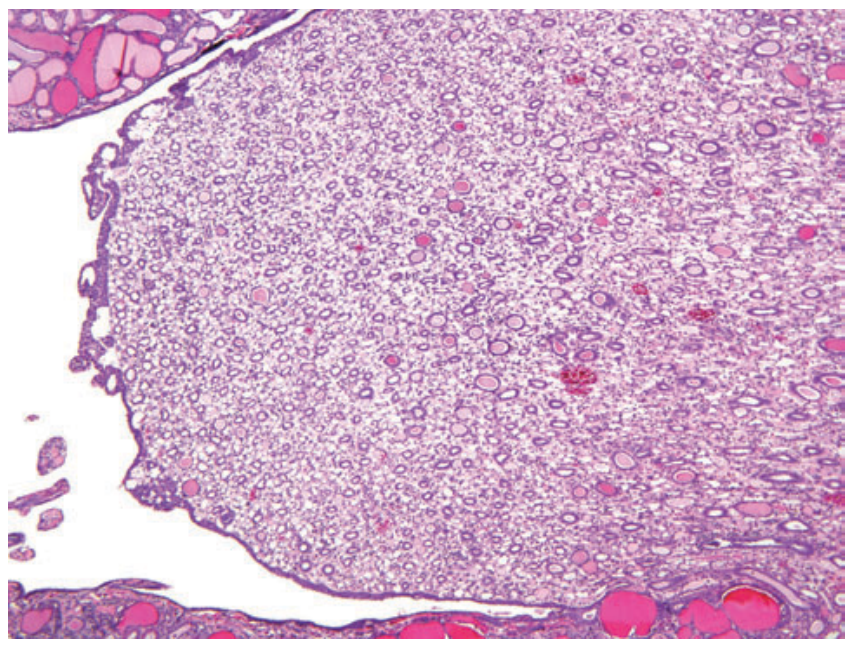

Fig. 4. Edematous and hyperplastic outgrowths of epithelium lining the renal papilla associated with CPN. H\&E. $\times 6.4$.

as part of advanced $\mathrm{CPN}$ and not diagnosed separately.

Aside from tangential sections or cell proliferation responses due to the presence of inflammatory cells, many of these CPN tubule profiles contained focal to segmental accumulation of tubule lining cells which exceeded more than a single cell layer (multicellular layering) within the confines of the tubule (Fig. 5) or in some cases a dilated tubule (Fig. 6). These tubules, in general, were surrounded by a thickened basement membrane and contained cells morphologically similar to adjacent CPN lining cells. Even though in a few cases, these tubules were solitary and larger than some of the other adjacent regenerative tubules, their irregular outline and thickened basement membrane helped distinguish them from preneoplastic tubule hyperplasia. Tangential sections through these larger CPN tubule profiles often had a "plaque-like" appearance which required careful examination to aid in discriminating this appearance from ATH (Fig. 7). These "plaque-like" structures lacked the 


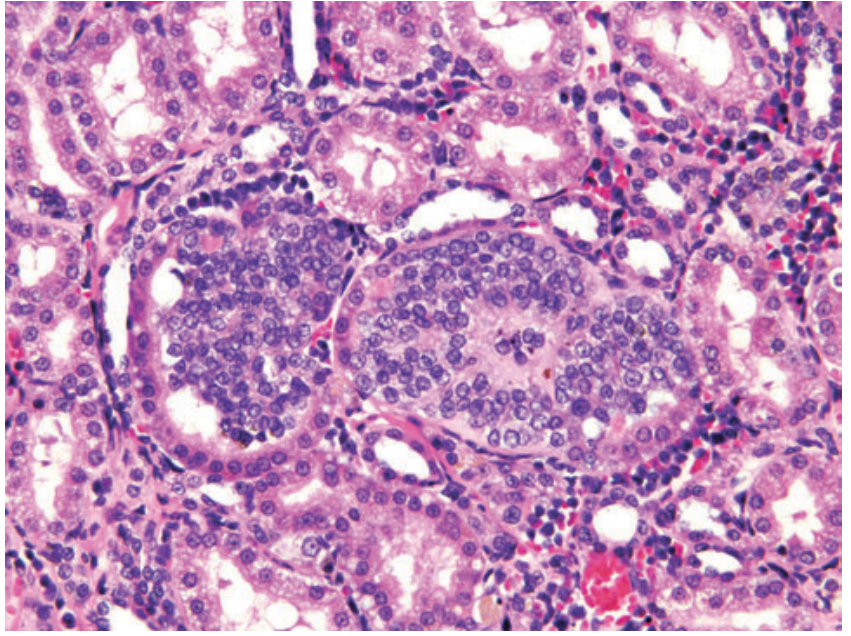

Fig. 5. Intratubular "cluster" of tubule epithelial cells with small, bland nuclei and indistinct cytoplasm. These cell proliferations occur near tubule bends and are usually noted in cases of moderate to marked CPN. H\&E. $\times 40$.

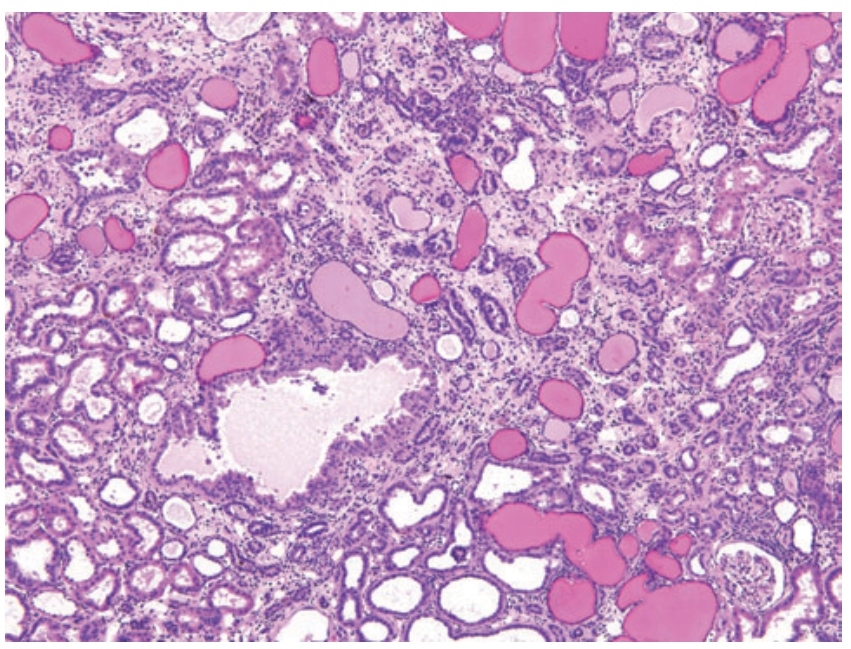

Fig. 6. Dilated tubule lined by multicellular epithelium. The presence of dilated tubules are common in moderate to marked cases of CPN. H\&E. $\times 10$.

high proliferative rate demonstrated by PCNA in $\mathrm{ATH}^{36}$.

The diagnosis of ATH within the context of marked to end-stage CPN should be made from the standpoint of several histological criteria. Guidelines for the diagnosis of ATH are presented in Table 1. Perhaps one of the most reliable criteria is the presence of fibroblasts which are seen encircling the ATH tubule suggesting early expansile growth of the lesion (Fig. 8). ATH may either be graded or not graded, however, in any grading scheme, the most severe lesion should be regarded as a borderline lesion with renal tubule adenoma.

\section{Evidence of CPN Associated Renal Tumorigenesis}

Although no definitive study has been designed or conducted to test the hypothesis of direct CPN associated

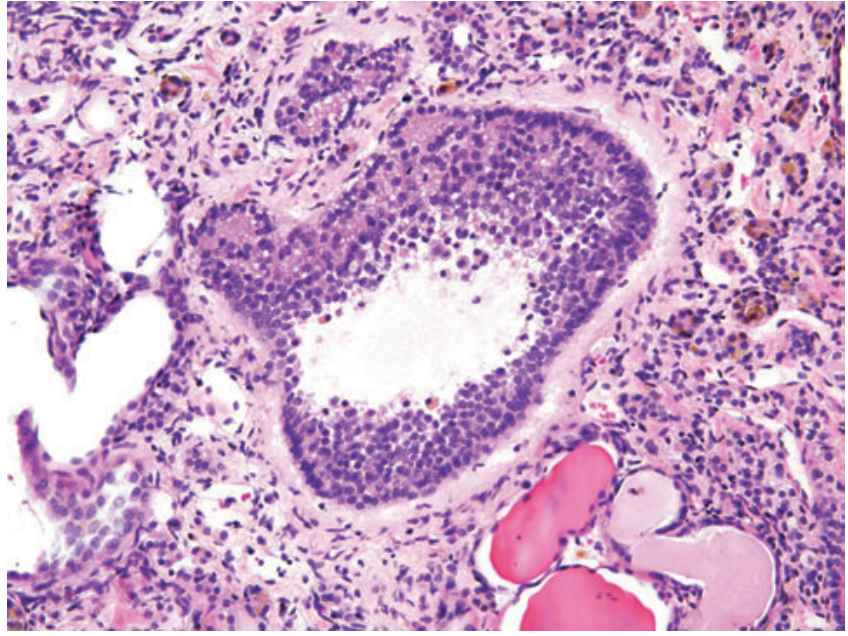

Fig. 7. "Plaque-like" tubule which is present in area of marked CPN. The tubule is surrounded by a thickened basement membrane with tangential appearance of multicellular epithelium. H\&E. $\times 25$.

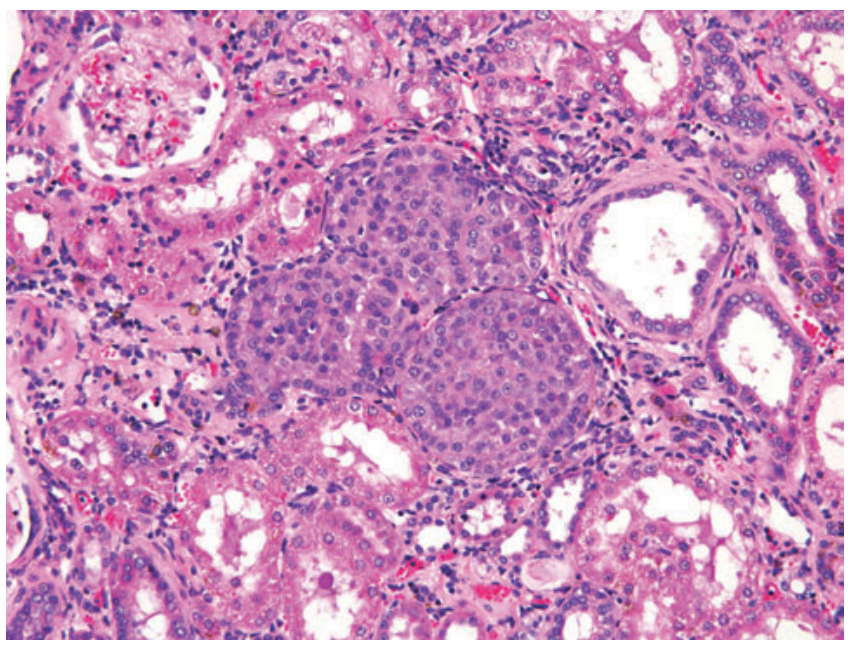

Fig. 8. Atypical tubule hyperplasia (ATH) which illustrates the characteristic basophilic staining of the hyperplastic epithelial cells and expansive nature of the lesion characterized by the presence of flattened fibroblastic cells encircling the margin of the hyperplastic tubule. The integrity of the tubule profile appears intact and there is no capillary in growth into the lesion. H\&E. $\times 25$.

renal tumorigenesis in rodents, there is a body of scientific evidence that strongly suggests that there is a relationship of CPN to renal carcinogenesis. It is doubtful if a study could be specifically designed to unequivocally prove this hypothesis so we are left with the information we have to date and hope new investigations will lead to further support of this hypothesis.

Over the years, several investigators have remarked on observations from various studies that there appeared to be a relationship between CPN and the development or risk of tubule neoplasms ${ }^{37-39}$. However, Dietrich and Swenberg noted that in some National Toxicology Program (NTP) 
Table 1. Main Criteria of Atypical Tubule Hyperplasia in Advanced $\mathrm{CPN}$

Proliferation of a single tubule (usually solid)

Thickened CPN-associated basement membrane usually absent

Cytoplasm and cell margins well developed

Basophilic staining with glassy sheen

Expansive lesion with fibroblasts encircling lesion (margination)

Table 2. Criteria of CPN Associated Tumorigenesis

Slight but usually statistical increase in renal tubule tumors

Exacerbation of CPN to advanced degrees of severity at doses associated with tumor increase

Tumors are usually adenomas (typically basophilic) which are often of small size or borderline with ATH

Absence of any cellular alterations indicative of chemical toxicity in parenchyma that is not involved in the $\mathrm{CPN}$ process

bioassay studies, which enhanced CPN, tumors were not increased $^{38}$. This could have been due to CPN not being exacerbated to a severe enough stage. In one study conducted to specifically investigate this relationship, the NTP data base was reviewed for the severity of CPN and the presence of renal tumors in control tumor- bearing F344 male rats with aged-matched control nontumor- bearing animals ${ }^{40}$. A slight, but statistically significant increase in the severity of CPN was noted in rats with renal tumors.

Determining the relationship of $\mathrm{CPN}$ and tumor development between control and chemically exposed animals within studies is difficult. For instance, some nongenotoxic chemicals exacerbate $\mathrm{CPN}$ and result in tumors ${ }^{41}$. However, several of these chemicals also demonstrate nephrotoxicity and, therefore, may be influencing tumorigenesis by other mechanisms ${ }^{35}$. Hydroquinone, one such chemical, was reported to exacerbate CPN and resulted in increased numbers of tubule adenomas $^{23}$. During the investigation into a possible mode of action, a treatment- associated, statistically significant increased severity grade of CPN in animals with ATH or adenomas was observed. In addition, the ATH and adenomas occurred in areas of severe to end-stage CPN.

Subsequently, two additional investigational studies utilizing the NTP bioassay study results of ethyl benzene and quercetin attempted to determine a mode of action of a small number of renal tumors in animals with exacerbated $\mathrm{CPN}^{24,25}$. These investigations used an expanded scale of CPN severity grade to re-evaluate affected kidneys and determined the distribution of tumors in relationship to the severity of CPN. Both of these investigations supported the role of exacerbated $\mathrm{CPN}$ as a mode of action underlying the development of renal tumors. Recently, a proposed set of criteria for recognizing a possible role of CPN-associated tumorigenesis from preclinical studies has been published ${ }^{3}$. These criteria are abstracted in Table 2.

The pathogenesis of $\mathrm{CPN}$-associated tumorigenesis is most likely multi-factorial and complex. Cellular proliferation plays a role in tissue regeneration and carcinogenesis ${ }^{42,43}$. Therefore, several of the proposed mechanisms suggested for CPN tumorigenesis include promotion by indirect secondary mechanisms facilitating the clonal expansion of altered cells, increasing the spontaneous error rate in DNA replication or decreasing the time for fixation of mutational events ${ }^{23}$.

The question of the importance of CPN tumorigenesis and human risk assessment has been examined by Hard and $\mathrm{Kahn}^{3}$. In their paper, they looked at several of the leading causes of chronic renal disease in humans (ie: hypertensive, diabetic and post-infection nephropathies) and compared these conditions to $\mathrm{CPN}$ of rodents. Additionally they compared the age progression, clinical pathology parameters such as proteinuria, and diet modifications of CPN with the same human renal diseases. From their extensive review they could not find any human disease counterpart which was similar to rodent CPN. Therefore, they concluded that renal tubule tumors linked to CPN by chemical exacerbation in a rodent preclinical study have no relevance for extrapolation in human risk assessment.

In rodent preclinical studies, a number of rodent specific mechanisms of toxicity/carcinogenesis have been shown to have little to no relevance for human risk assessment ${ }^{44}$. It would appear that the accumulating body of evidence seems to support the postulated "Mode of Action" of CPN tumorigenesis within the framework for human relevance analysis ${ }^{45}$.

\section{References}

1. Gray JE. Chronic progressive nephrosis in the albino rat. CRC Crit Rev Toxicol. 5: 115-144. 1977.

2. Barthold SW. Chronic progressive nephropathy. Rat In: Urinary System, $2^{\text {nd }}$ ed. TC Jones, GC Hard and U Mohr (eds). Springer-Verlag, Berlin. 228-233. 1989.

3. Hard GC and Khan KN. A contemporary overview of chronic progressive nephropathy in the laboratory rat, and its significance for human risk assessment. Toxicol Pathol. 32: 171-180. 2004.

4. Goldstein RS, Tarloff JB and Hook JB. Age-related nephropathy in laboratory rats. FASEB. J2: 2241-2251. 1988.

5. Baylis C. Age-dependent glomerular changes in the rat. Dissociation between glomerular injury and both glomerular hypertension and hypertrophy. Male gender as a primary risk factor. J Clin Invest. 94: 1823-1829. 1994.

6. Gray JE, Van Zwieten MJ and Hollander CF. Early light microscopic changes in chronic progressive nephrosis in several strains of aging laboratory rats. J Gerontol. 27: 142150. 1982.

7. Peter CP, Burek JD and van Zwieten MJ. Spontaneous nephropathies in rats. Toxicol Pathol. 14: 91-100. 1986.

8. Solleveld HA and Boorman GA. Spontaneous renal lesions in five rat strains. Toxicol Pathol. 14: 168-174. 1986.

9. Percy DH and Barthold SW. Pathology of Laboratory Rodents and Rabbits. Iowa State University Press, Ames. 149-151. 2001.

10. Palm M. The incidence of chronic progressive nephrosis in young Sprague-Dawley rats from two different breeders. 
Lab Anim. 32: 477-482. 1998

11. Owen RA and Haywood R. Age-related variations in renal structure and function in Sprague-Dawley rats. Toxicol Pathol. 14: 158-167. 1986.

12. Abrass CK. The nature of chronic progressive nephropathy in aging rats. Adv Renal Replacement Therapy. 7: 4-10. 2000.

13. Rao GN. Diet and kidney diseases in rats. Toxicol Pathol. 30: 651-656. 2002.

14. Rao GN, Morris RW and Seely JC. Beneficial effects of NTP-2000 diet on growth, survival and kidney and heart diseases of Fischer 344 rats in chronic studies. Toxicol Sci. 63: 245-255. 2001.

15. Everitt AV, Porter BD and Barnard DJ. Effects of caloric intake and dietary composition on the development of proteinuria, age-associated renal disease and longevity in the male rat. Gerontology. 28: 168-175. 1982.

16. Masoro EJ, Iwasaki K, Gleiser CA, McMahan CA, Seo EJ, and $\mathrm{Yu}$ BP. Dietary modulation of the progression of nephropathy in aging rats: an evaluation of the importance of protein. Am J Clin Nutr. 49: 1217-1227. 1989.

17. Short BG and Goldstein RS. Nonneoplastic lesions in the kidney. In: Pathology of the Aging Rat. U Mohr, DL Dungworth, CC Capen (eds). ILSI Press, Washington, DC. 211-219. 1992.

18. Keenan KP, Coleman JB, McCoy CL, Hoe C-M, Soper KA and Laroque $\mathrm{P}$. Chronic nephropathy in ad libitum overfed Sprague-Dawley rats and its early attenuation by increasing degrees of dietary (caloric) restriction to control growth. Toxicol Pathol. 28: 788-798. 2000.

19. Sworn MJ and Fox M. Renal age changes in the rat compared with human renal senescence. An autoradiographic study. Invest Urol. 12: 140-145. 1974.

20. Konishi N and Ward JM. Increased levels of DNA synthesis in hyperplastic renal tubules of aging nephropathy in female F344/NCA rats. Vet Pathol. 26: 6-10. 1989.

21. Short BG, Burnett VL and Swenberg JA. Elevated proliferation of proximal tubule cells and localization of accumulated alpha 2-microglobulin in F344 rats during chronic exposure to unleaded gasoline or 2,2,4trimethylpentane. Toxicol Appl Pharmacol. 101: 414-431. 1989.

22. Hard GC and Seely JC. Recommendations for the interpretation of renal tubule proliferative lesions occurring in rat kidneys with advanced chronic progressive nephropathy (CPN). Toxicol Pathol. 33: 641-649. 2005.

23. Hard GC, Whysner J, English JC, Zang E and Williams GM. Relationship of hydroquinone-associated rat renal tumors with spontaneous chronic progressive nephropathy. Toxicol Pathol. 25: 132-143. 1997.

24. Hard GC. Significance of the renal effects of ethyl benzene in rodents for assessing human carcinogenic risk. Toxicol Sci. 69: 30-41. 2002.

25. Hard GC, Seely JC, Betz LJ and Hayashi S. Re-evaluation of the kidney tumors and renal histopathology occurring in a 2year carcinogenicity bioassay of quercetin. Food Chem Toxicol. 45: 600-608. 2007.

26. Montgomery CA and Seely JC. Kidney. In: Pathology of the Fischer Rat. Reference and Atlas. GA Boorman, SL Eustis, MR Elwell, CA Montgomery and WF Mackenzie (eds). Academic Press, San Diego. 127-153. 1990.

27. Greaves P. Urinary tract. In: Histopathology of Preclinical
Toxicity Studies. (3rd ed). Elsevier, Amsterdam. 581-584. 2007.

28. Thudium D, Adams D, Sellers T, Rehm S, Ennulat D and Schwartz L. Urinary albumin as a sensitive marker for nephropathy in aged male rats. Vet Pathol. 38: 571. 2001.

29. Gray JE, Weaver RM and Purmalis A. Ultrastructural observations of chronic progressive nephrosis in the Sprague-Dawley rat. Vet Pathol. 11: 153-164. 1974.

30. Nakatsuji S, Yamate J and Sakuma S. Relationship between vimentin expressing renal tubules and interstitial fibrosis in chronic progressive nephropathy in aged rats. Virchows Arch. 433: 359-367. 1998.

31. Nakatsuji S, Yamate J and Sakuma S. Macrophages, myofibroblasts, and extracellular matrix accumulation in interstitial fibrosis of chronic progressive nephropathy in aged rats. Vet Pathol. 35: 352-360. 1998.

32. Ward JM and Reznik-Schuller H. Morphological and histochemical characteristics of pigments in aging F344 rats. Vet Pathol. 17: 678-685. 1980.

33. Hard GC, Alden CL, Stula EF and Trump BF. Proliferative lesions of the kidney in rats. In: Guides for Toxicologic Pathology. STP/ARP/AFIP, Washington, DC. 1995.

34. Hardisty JF. Personal communication. 2007.

35. Hard, GC. Mechanisms of chemically induced renal carcinogenesis in the laboratory rodent. Toxicol Pathol. 26: 104-112. 1998.

36. Hard GC and Seely JC. Histological investigation of diagnostically challenging tubule profiles in advanced chronic progressive nephropathy (CPN) in the Fischer 344 rat. Toxicol Pathol. 34: 941-948. 2006.

37. Lipsky MM and Trump BF. Chemically induced renal epithelial neoplasia in experimental animals. Int Rev Exp Pathol. 30: 357-383. 1988.

38. Dietrich DR and Swenberg JA. Preneoplastic lesions in rodent kidney induced spontaneously or by non-genotoxic agents: predictive nature and comparison of lesions induced by genotoxic carcinogens. Mutation Res. 248: 239-260. 1991.

39. Anver MR, Cohen BJ, Lattuada CSP and Foster SJ. Ageassociated lesions in barrier-reared male Sprague-Dawley rats: A comparison between Hap: (SD) and Cal:COBS ${ }^{\circledR}$ SD stocks. Exp Aging Res. 8: 3-24. 1982.

40. Seely JC, Haseman JK, Nyska A, Wolf DC, Everitt JI and Hailey JR. The effect of chronic progressive nephropathy on the incidence of renal tubule cell neoplasms in control male F344 rats. Toxicol Pathol. 30: 681-686. 2002.

41. Khan KNM and Alden CL. Kidney. In: Handbook of Toxicologic Pathology, $2^{\text {nd }}$ ed. WM Hascheck, CG Rousseaux and MA Walling (eds). Academic Press, San Diego, 255-335. 2002.

42. Tomatis L. Cell proliferation and carcinogenesis. A brief history and current view based on IARC Workshop report. Environ Healh Perspect. 100 (Suppl.5): 149-152. 1993.

43. Cohen SM. Role of cell proliferation in regenerative and neoplastic disease. Toxicol Letter. 82/83: 15-21. 1995.

44. Ward JM. The two-year rodent carcinogenesis bioassaywill it survive. J Toxicol Pathol. 20: 13-19. 2007.

45. Meek ME, Bucher JR, Cohen SM, Dellarco V, Hill RN, Lehman-Mckeeman LD, Longfellow DG, Pastoor T, Seed J and Patton DE. A framework for human relevance analysis of information on carcinogenic modes of action. Crit Rev Toxicol. 33: 591-653. 2003. 